\title{
UN ANTAGONISMO LARVADO. CONFLICTIVIDAD Y REYERTAS ENTRE LOS COMERCIANTES RIOPLATENSES (1789-1794)
}

\author{
MARIANO MARTIN SCHLEZ \\ Universidad Nacional del Sur/CONICET (Argentina)
}

\section{RESUMO}

$\mathrm{O}$ artigo tem como objetivo analisar os confrontos entre os comerciantes do Rio de la Plata, antes do estabelecimento do Consulado de Buenos Aires (1794), a fim de compreender a natureza social dos lados opostos e os fundamentos materiais de seu comportamento . Para fazer isso, vamos rever a disputa levantada em Buenos Aires para a cobrança de imposto sobre as vendas no varejo, o confronto sobre a tentativa (falha) para substituir a Junta Comercial de Buenos Aires (1789) e os conflitos entre os comerciantes no Cabildo Buenos Aires.

PALAVRAS-CHAVE: Comerciantes; Río de la Plata; Império Espanhol.

\section{ABSTRACT}

The article aims to analyze the confrontations between traders in the Rio de la Plata, previous to establishment of the Consulate of Buenos Aires (1794), in order to understand the social nature of the opposing sides and the material foundations of their behavior. To do this we will review the dispute given rise in Buenos Aires for the collection of alcabala on second sale, the confrontation around the attempt (failed) to replace the agents of trade of Buenos Aires (1789), and conflicts between traders in the Cabildo Buenos Aires.

KEYWORDS: Merchants; River Plate; Spanish Empire. 
En los últimos tiempos, se han desarrollado notablemente los estudios dedicados a la actuación de los comerciantes en la modernidad o, más exactamente, en la transición del feudalismo al capitalismo ${ }^{1}$. Dicho crecimiento no sólo tuvo como protagonistas el análisis de los mercados europeos, sino que también incluyó al continente americano, en general, y al Río de la Plata, en particular².

Buena parte de las investigaciones coinciden en categorizar a los comerciantes coloniales como integrantes de una élite que, dependiendo de su contexto, mutó al calor de la crisis de fines del siglo XVIII y principios del XIX ${ }^{3}$. No obstante, luego de evidenciarse una serie de límites y problemas surgidos del marco teórico empleado ${ }^{4}$, los últimos años han sido testigos de la revitalización de la categoría de clase social para el análisis del capital mercantil colonial. ${ }^{5}$ Su utilización ha evidenciado los límites de una mirada concentrada en aspectos puramente subjetivos, señalando las virtudes de una mirada que dé cuenta de los aspectos materiales y sociales, para comprender el derrotero de aquellos mercaderes ${ }^{6}$.

En ese sentido, este artículo tiene por objetivo analizar los enfrentamientos entre comerciantes rioplatenses, previos al establecimiento del Consulado de Buenos Aires (1794), con el objetivo de comprender la naturaleza

${ }^{1}$ La bibliografía es amplia y extensa, por lo que nos conformamos con citar los trabajos más recientes, que dan cuenta de la vigencia del problema. Véase CRESPO SOLANA, Ana (Coord.). Comunidades transnacionales: colonias de mercaderes extranjeros en el Mundo Atlántico (15001830). Madrid: Ediciones Doce Calles, 2010; LOBATO, I.; OLIVA, J. M. (Ed.). El sistema comercial español en la economía mundial (siglos XVII-XVIII). Huelva: Universidad de Huelva, 2013; HERRERO, María Dolores. El mundo de los negocios de Indias. Madrid: CSIC-Universidad de Sevilla-Diputación de Sevilla, 2013.

2 LLORCA-JAÑA, Manuel. British merchants in new markets: the case of Wylie and Hancock in Brazil and the River Plate, c.1808-1820. Journal of Imperial and Commonwealth History, Volume 44, 2014; DALLA CORTE, Gabriela. Vida i mort d' una aventura al Riu de la Plata. Jaime Alsina y Verjés, 1770-1836. Barcelona: Publicacions de L'Abadia de Montserrat, 2000; JUMAR, Fernando. Comerciantes en Río de la Plata pre-virreinal. El caso de Domingo de Basavilbaso. En: Anuario del Instituto de Historia Argentina. Facultad de Humanidades y Ciencias de la Educación, UNLP, No 11, 2011.

3 NAVARRO GARCÍA, Luis (coord.). Élites urbanas en Hispanoamérica (De la conquista a la independencia). Sevilla: Universidad de Sevilla, 2005; MATA DE LÓPEZ, Sara. La conformación de las élites a fines de la colonia, Comerciantes y hacendados en la sociedad de Salta, Argentina. Colonial Latin American Historical Review, 9:2, Nuevo México, 2000; MAZZEO, Cristina Ana (Dir.). Los comerciantes limeños a finales del siglo XVIII, capacidad y cohesión de una élite 1750-1825. Lima: Universidad de Lima, 1999.

${ }^{4}$ BÜSCHGES, Christian; LANGUE, Frédérique. ¿Las élites de la América española, del éxito historiográfico al callejón interpretativo? Reconsideraciones. En: BÜSCHGES, Christian; LANGUE, Frédérique (coords.). Excluir para ser. Procesos identitarios y fronteras sociales en la América Hispánica (XVII-XVIII), Frankfurt-Madrid: Vervuert Verlag-Iberoamericana, 2005.

${ }^{5}$ HARARI, Fabián. Hacendados en Armas. El Cuerpo de Patricios de las Invasiones Inglesas a la Revolución (1806-1810). Buenos Aires: Ediciones ryr, 2009; VOVELLE, Michel, CHUST, Manuel y SERRANO ORTEGA, José A. Escarapelas y coronas. Las revoluciones continentales en América y Europa, 1776-1835. Venezuela: Editorial ALFA, 2012; DAVIDSON, Neil. Transformar el mundo. Revoluciones burguesas y revolución social. Barcelona: Ediciones Pasado y Presente, 2013.

${ }^{6}$ SCHLEZ, Mariano. Diego de Agüero y los fundamentos económicos de la clase dominante en el Río de la Plata tardo colonial (1770-1810). En: GARCÍA DE LEÓN, Antonio; JÁUREGUI, Luis; SERRANO ORTEGA, José A. (Ed.). La Corona en llamas. Cuestiones económicas y sociales en las independencias iberoamericanas. Castellón: Universitat Jaume I, 2010; BRENNER, Robert. Mercaderes y revolución. Madrid: Akal, 2011. 
social de los bandos enfrentados, así como los fundamentos materiales de su comportamiento ${ }^{7}$. Para ello, revisaremos el conflicto suscitado en Buenos Aires por el cobro de la alcabala de reventa, el enfrentamiento en torno al intento (fallido) por reemplazar a los apoderados del comercio porteño en 1789, y los conflictos entre comerciantes desarrollados, ese mismo año, en el Cabildo de Buenos Aires.

La documentación trabajada proviene tanto del Archivo General de la Nación Argentina (AGN), fundamentalmente actas de Juntas de Comercio y del Cabildo, expedientes del Consulado y correspondencia mercantil; del Museo Histórico Nacional de Uruguay (MHN); y del Archivo General de Indias de Sevilla (AGI), particularmente documentos del repositorio Buenos Aires y Consulados.

\section{El conflicto por la alcabala de reventa}

Desde mediados de la década de 1750, los comerciantes rioplatenses (es decir, aquellos que actuaron en la región del Río de la Plata, Buenos Aires y Montevideo, fundamentalmente) Ilevaron adelante un frente común para enfrentar los privilegios de los mercaderes radicados en Lima ${ }^{8}$. Lo hicieron realizando numerosas Juntas de Comercio, que no sólo tenían el objetivo de obtener reformas que dieran a Buenos Aires un status legal similar al de Lima, sino que también buscaron autorización para instaurar un Consulado propio ${ }^{9}$.

El éxito de sus reclamos no detuvo otro proceso paralelo, de profundización de una serie de diferencias (materiales, sociales y políticas) que comenzaban a tomar la forma de facciones y grupos enfrentados. Uno de estos primeros conflictos se dio entre los "tenderos", es decir, entre los poseedores de tiendas de comercio de venta al público, con motivo del cobro de la alcabala de reventa o segunda venta.

A fines de 1788, en la casa de Manuel Rodríguez de la Vega, los comerciantes dueños y tenedores de tiendas, que vendían "al menudeo", es

\footnotetext{
${ }^{7}$ Unos pocos trabajos han estudiado este tema y período, aunque con el objetivo de analizar el programa común de los comerciantes, relegando una explicación en torno a sus disputas. Al respecto, véase KRASELSKY, Javier. De las Juntas de Comercio al Consulado. Los comerciantes rioplatenses y sus estrategias corporativas, 1779-1794. En: Anuario de Estudios Americanos, 64, 2, julio-diciembre, pp. 145-170, Sevilla, 2007; MARILUZ URQUIJO, José María. Solidaridades y antagonismos de los comerciantes de Buenos Aires a mediados del setecientos. Investigaciones y Ensayos. No 35, 1987 y SOCOLOW, Susan. Economic Activities of the Porteño Merchants: The Viceregal Period. En: The Hispanic American Historical Review, Vol. 55, No. 1, Feb., 1975, pp. 124; TJARKS, Germán. El consulado de Buenos Aires y sus proyecciones en la historia del Río de la Plata. Buenos Aires: UBA-FFyL, 1962.

${ }^{8}$ SCHLEZ, Mariano. Comercio y sociedad en el Virreinato del Río de la Plata (1770-1820). En: CAVA MESA, Begoña (Coord.). América en la Memoria: Conmemoraciones y Reencuentros. Tomo II. Bilbao: Asociación Española de Americanistas - Universidad de Deusto, 2013, pp. 427-446.

9 SCHLEZ, Mariano. Buenos Aires versus Lima. La estrategia reformista de los comerciantes porteños (1770-1794). En: Illapa. Revista Latinoamericana de Ciencias Sociales. Lima: Año 4, No 9, abril de 2014.
} 
decir, al por menor, en la ciudad de Buenos Aires, eligieron a José Romero del Villar y Roque Burrigorri como responsables del cobro de la alcabala de segunda venta. Dicho impuesto lo abonaban todos los comerciantes de Indias por sus ventas por menor, en base a un monto fijado de antemano por un funcionario estatal, conforme al estado del comercio. Su cobro había sido delegado por la Corona, en los comerciantes, quienes podían organizar ellos mismos los tiempos y formas de los pagos. Luego de que la burocracia haya fijado el monto, nombraban en Junta a dos "repartidores de alcabala" quienes, de acuerdo a la riqueza de cada uno, fijaban la cuota que le correspondía pagar. Toda duda o recusación se sometía al Administrador de la Aduana o a los funcionarios de la Real Hacienda que designara el Virrey a tal efecto ${ }^{10}$.

Dado que se trataba de un impuesto al comercio al por menor, una parte considerable de los grandes comerciantes porteños no debían abonarlo, en tanto desarrollaban una actividad comercial exclusivamente al por mayor. No obstante, veremos que no permanecían ajenos al problema de su cobro, dado que los enfrentamientos entre tenderos llevaron a su arbitraje.

En 1793, veintiún comerciantes, entre los que se encontraban Esteban Villanueva, Pedro Duval, Juan Antonio Zelaya y Tomás Fernández, se quejaron de "la excesiva desproporción" con que los comisionados habían distribuido el pago de la alcabala, por lo que solicitaron se suspenda su cobro y se convoque a Junta de Comercio para debatir este punto. Otros protestaron por ser incluidos en semejante impuesto sin siquiera ser vendedores al menudeo, como Juan Esteban de Anchorena. En la Junta, se formalizó la denuncia de que el reparto estaba

en todas sus partes mal hecho. Así por la monstruosa desproporción que han notado respecto de unos individuos a otros, como por haber comprendido en él a varios individuos que no deben contribuir cosa alguna, por haber pagado en la Real Aduana la alcabala de primera venta, al tiempo de la introducción de los efectos, y otros que no se han tenido en consideración.

Se planteaba, entonces, que debía "anularse dicho repartimiento y formase otro nuevo por personas de conocimiento e inteligencia, para que cada uno señale lo que deba contribuir justamente"11.

Sin embargo, no todos pensaban del mismo modo: en la Junta, se formaron "dos encontrados bandos (...) unos defendiendo la regulación, otros contradiciéndola"12. Buscando resolver esta diferencia, en la que sus

\footnotetext{
${ }^{10}$ Luego de la instauración del Consulado, sus consiliarios debían revisar los padrones, recaudar las cantidades que traían los cobradores, informar sobre los casos en que no era posible la recaudación por disputas o injusticia y, finalmente, formar una cuenta de las cantidades recibidas y de los fondos entregados al tesorero del Tribunal. El cuerpo utilizaba todo tipo de recursos para, ante los reclamos del Virrey, dilatar el pago (hasta argumentar que las calles estaban embarradas por la lluvia y no se podía cobrar). Para realizar los cobros, todos los años se elegían representantes que debían realizar una "regulación impositiva", que establecía los montos y las fechas para realizar los pagos destinados a la Corona. Véase TJARKS, op. cit., pp. 479-480.

${ }_{11}$ AGN, Sala IX, Consulado, Expedientes, 1771-1793, Expediente No 13, 4-7-3.

${ }^{12}$ AGN, Sala IX, Consulado, Expedientes, 1771-1793, Expediente № 26, 4-7-3.
} 
participantes no podían ser juez y parte, los comerciantes Esteban de Avellaneda y Mariano Zabaleta solicitaron al Virrey que nombrase "a personas independientes, y de probidad conocida", proponiendo a los comerciantes "por mayor" Argibel, Agüero, Álzaga, Necochea, Ugarte, Anchorena, Ezcurra, Gaona, Castañón, Sánchez de Velazco, Duval o Flotat, quienes, además de poseer las citadas características, "no les toca en lo menor la regulación" por no poseer tiendas al menudeo ${ }^{13}$. Por lo que, en marzo de 1793, el Virrey delegó la tarea de "examinar, corregir y enmendar la regulación de la alcabala de reventa correspondientes a los años 89, 90, 91 y 92" a Diego Agüero, Martín de Álzaga, Joaquín de Arana y Casimiro Francisco Necochea ${ }^{14}$. Su corrección trajo algunas novedades: de los 255 comerciantes, 14 fueron eximidos (la mayoría por tratarse de grandes comerciantes, como Juan Antonio Zelaya, Juan Esteban de Anchorena y Pedro Duval, que abonaban alcabala de primera venta), 9 recibieron rebajas (entre ellos, el primer denunciante, Esteban de Avellaneda) y a 37 se le aumentaron (quienes, de acuerdo a su giro, podían realizar mayores aportes, como Francisco de Tellechea, Cristóbal de Aguirre, Juan Antonio de Santa Coloma e Isidro José Balbastro $)^{15}$.

Sin embargo, esta regulación de "personas independientes" no trajo la paz: los principales perjudicados (como Ramón Román Díaz, Juan Antonio de Santa Coloma, Francisco de Tellechea y Juan José de Arana, entre otros) la impugnaron, defendiendo la anterior regulación. Plantearon "que las personas últimamente nombradas no tienen inteligencia en la materia del manejo de tiendas" y que "su operación ha sido toda desproporcionada y sin guardar equidad alguna", considerando erróneo eximir del pago a tantos comerciantes "con el pretexto de que sus ventas son de primera mano" y rehusándose a pagar, a pesar de las advertencias de la Real Aduana. Tan poderosa fue su protesta que, en mayo de 1794, el fiscal resolvió dejar de lado esta segunda regulación y cobrar según la anterior, aunque manteniéndole la excepción a Esteban de Avellaneda.

\footnotetext{
${ }^{13}$ Una "razón de los individuos dueños y encargados de tiendas de esta ciudad", las regulaciones de 1787-1792 y la declaración de Esteban de Avellaneda nos permiten confirmar que Diego de Agüero no poseía tiendas al menudeo en Buenos Aires (al igual que Álzaga, Necochea, Ugarte, Anchorena, Ezcurra, Gaona, Castañón, Sánchez de Velazco y Flotat). Eran a la vez importadores y poseedores de tiendas, entre otros, Francisco de Tellechea, Julián del Molino Torres, José Antonio Capdevila, Francisco Escalada, Isidro José Balbastro, Esteban Villanueva, Tomás de Balenzategui, Manuel Ventura de Haedo, Jayme Alsina y Verjés, Tomás Fernández, Juan Antonio de Santa Coloma, Cristóbal de Aguirre, Miguel de Azcuénaga, Manuel Ortiz de Basualdo y José Martínez de Hoz. AGN, Sala IX, Consulado, Expedientes, 1771-1793, Expedientes № 12, 13 y 26, 4-7-3.

${ }^{14}$ En un primer momento había sido elegido Luis de Gardeazábal, pero fue recusado por Avellaneda y Zabaleta por ser pariente de uno de los beneficiados por la regulación, Juan José Arana. En su lugar solicitan se nombre a Juan José Lezica, pero éste se excusa, al igual que Bernardo Gregorio de las Heras. También es recusado Gaspar de Santa Coloma, por estar asociado a su sobrino, Juan Antonio, en el mantenimiento de una tienda. AGN, Sala IX, Consulado, Expedientes, 1771-1793, Expediente $\mathrm{N}^{\circ} 26,4-7-3$.

${ }^{15}$ AGN, Sala IX, Consulado, Expedientes, 1771-1793, Expediente № 26, 4-7-3.
} 
Luego de este nuevo conflicto, el Virrey ordenó nombrar nuevos comisionados para recaudar la alcabala de 1793, planteando que deberían aprobar su regulación en Junta de Comercio, para que no vuelva a ocurrir el mismo incidente. En febrero de 1794, en la casa de Martín de Sarratea, fueron elegidos Ramón Román Díaz y José de Oyuela. Pero luego de instituirse, en 1794, el Consulado se encargó, gratuitamente, de cobrar la alcabala de reventa. Al tomar esta tarea, elevó una representación al Virrey solicitando que no aumente el derecho y se mantenga dentro de los límites de lo percibido en años anteriores. Fue así como el Virrey decretó que la alcabala "se cobre a razón de ocho mil pesos al año, dejando el modo y forma de ejecutarlo a este Real Consulado". La Junta determinó que Díaz y Oyuela mantuvieran la responsabilidad del cobro, pero nombró a Diego de Agüero y Cristóbal de Aguirre para atender las cuestiones de competencia que surgieran, otorgándosele "entera facultad para el caso, hasta que se haga efectivo el pago de la cantidad que a cada uno de los contribuyentes corresponde". Nuevamente, uno de los que resistió al pago fue Esteban Villanueva, que exigía que los repartidores sean elegidos en Junta de Comercio, rechazando la autoridad del Consulado. Pero el Tribunal dispuso cortar toda discusión y ratificó a Agüero y a Aguirre en sus tareas hasta que completaran la recaudación, dejando a los peticionantes el derecho a apelar por el exceso en la distribución. En enero de 1795, los comisionados presentaron su regulación, que fue aprobada por Agüero y Aguirre, quienes delegan el cobro a Pedro González Cortina. El 10 de mayo de 1796, ambos presentaron un oficio con el expediente obrado sobre la regulación de alcabala de reventas de los años 1793 y 1794, dando por terminada la comisión encargada por el Tribunal ${ }^{16}$.

Este pequeño incidente prueba la existencia de diferencias, tanto materiales como gremiales, entre los comerciantes porteños. Aunque en este conflicto específico las alianzas tejidas no muestran un patrón claro y definido, más allá de la evidente divergencia de intereses entre mayoristas y minoristas, otro tipo de enfrentamientos que ocurrían en paralelo dan cuenta que no estamos frente a combates aleatorios ni coyunturales, sino frente a la conformación embrionaria de intereses orgánicos.

\section{Intento de remoción de los Apoderados del Comercio de Buenos Aires}

El 17 de octubre de 1789, un crecido número de comerciantes, entre los que se encontraban Diego de Agüero, Martín de Álzaga, Román Ramón Díaz, Jaime Alsina y Verjés, Juan José Lezica, Francisco Antonio de Escalada, Joaquín y Manuel de Arana, Casimiro Francisco de Necochea y Gaspar de Santa Coloma, solicitaron al Virrey la convocatoria a Junta de Comercio, con el fin de reemplazar a los apoderados Bernardo Sancho Larrea, que había regresado a

\footnotetext{
${ }^{16}$ AGN, Consulado de Buenos Aires, op. cit., p. 260; AGN, Sala IX, Consulado, Expedientes, 17941797, Expediente $\mathrm{N}^{\circ}$ 19, 4-7-4; TJARKS, op. cit., p. 480.
} 
España en 1786, y a Manuel Rodríguez de la Vega, cuya ancianidad le impedía cumplir sus funciones cabalmente ${ }^{17}$.

Autorizados por el Virrey, De la Vega y Sarratea citaron a Junta para el 20 de octubre, en casa del primero. Sin embargo, ese día, algunos plantearon que desconocían el motivo de la reunión, y otros se lamentaron de la escasa concurrencia, por lo que coincidieron en posponer la elección algunos días, para pensar a quién nombrar como apoderado y poder avisar a más comerciantes. ${ }^{18}$ Dado que los argumentos no son muy convincentes (era extraño que en una pequeña ciudad se desconociera algo tan elemental como los motivos de una convocatoria tan reducida, y asimismo, era regular que asistieran pocos comerciantes a las juntas), es probable que los presentes hayan intentado dilatar la votación por algún otro motivo, posiblemente por no existir acuerdo en torno a los apoderados que debían ser electos.

El día 24, finalmente, con 86 comerciantes presentes (aunque con algunas ausencias importantes, como la del mayorista Diego de Agüero), fueron elegidos Cristóbal de Aguirre, con 71 votos, y Miguel de Azcuénaga, con 37. Sin embargo, Casimiro Francisco de Necochea, Antonio García López, Luis de Gardeazábal, Juan de Salinas, Julián Barruro, Francisco Lezica, Saturnino Saraza y Andrés Lista, pidieron la nulidad de la Junta, denunciando la participación y el voto de comerciantes inhabilitados. Apelaron a los reglamentos de los Consulados de Sevilla, Bilbao y Lima, que dictaminaban que, para poder votar, era obligatorio ser comerciante matriculado, consignatario, dueño de casa o haber pagado avería ${ }^{19}$.

Probablemente influido por la protesta de tan renombrados mercaderes (Necochea era el apoderado del Consulado de Cádiz en Buenos Aires) ${ }^{20}$, Cristóbal de Aguirre rechazó el cargo, aludiendo sus numerosos cargos públicos, su precario estado de salud y el descuido de sus giros. Este hecho le permitió a los disconformes volver a solicitar al Virrey la anulación de la elección, incorporando a su argumentación la escasa concurrencia, que habría implicado la inasistencia de comerciantes "indispensables" (refiriéndose, probablemente, al mencionado Agüero). Desarrollaron su presentación denunciando que los comerciantes inhabilitados fueron llevados "para abultar y sacar la votación que llevaban premeditada", como Francisco González, quien habría sido un simple soldado,

\footnotetext{
${ }^{17}$ AGN, Sala IX, Comerciales, Legajo 14, Expediente 13, 30-9-9 y Consulado, Expedientes, 17711793, Expediente $N^{\circ} 14,4-7-3$.

${ }^{18}$ Asistieron ese día Diego de Agüero, Martín de Álzaga, Román Ramón Díaz, Tomás Fernández, Miguel González de Noriega, Joaquín de Arana, Francisco Ignacio de Ugarte, Gaspar de Santa Coloma, Isidro José Balbastro, José María Baquero, Antonio García López, Francisco Castañon, José Martínez de Hoz, Julián Hernández, Cristóbal Avecilla, Andrés de Lista, Tomás Insúa, Juan Agustín Videla, Juan Ángel de Molinuevo, Cecilio Sánchez de Velasco, Pedro González de Cueto, Juan Antonio de Zelaya, Manuel Rodríguez Mata, Pedro González Cortina, Ambrosio de Mier, Francisco Bosch, Félix Alvarez, José Fernández de Castro, Manuel Rodríguez de la Vega, Martín de Sarratea, Antonio Doldan, Francisco Antonio de Belautegui, Miguel Sancho Larrea, Juan Martín de Pueyrredón, Juan de Lagrava y Pablo Beruti.

${ }^{19}$ AGN, Sala IX, Comerciales, Legajo 14, Expediente 13, 30-9-9.

${ }^{20}$ AGI, Consulados, 339.
} 
Miguel de Larrea "actualmente demente como es público"21, José Montalvo y Andrés Fernández "dependientes de Ventura Llorente". Plantearon, entonces, que hubo "sujetos empeñados por Miguel de Azcuénaga y Cristóbal de Aguirre". Incluso señalaron que, en el recuento de votos, faltaba una boleta de sufragio, lo que confirmaría el fraude. Asimismo, aseguraron que los cargos que poseían ambos diputados electos (Azcuénaga era Alcalde de segundo voto del Cabildo y Aguirre, Regidor) eran incompatibles con este nuevo nombramiento. La solicitud finalizaba proponiendo, además de la anulación, una nueva elección donde participasen sólo comerciantes matriculados, exigiendo la confección de un listado con los sujetos acreditados para votar.

A ellos se enfrentaron una serie de comerciantes, representados por Francisco Ignacio de Ugarte, Agustín Antonio de Erezcano y Mariano Pérez de Saravia, que sostuvieron la elección realizada y solicitaron al Virrey su confirmación. En el documento relataron cómo se sucedieron los hechos. En primer lugar señalan que, a pesar de la protesta que antes de la votación interpuso Luis de Gardeazábal, los comerciantes decidieron votar. Sin embargo, luego de la elección, Gardeazábal, esta vez junto a otros siete comerciantes, y apelando a las ordenanzas de los Consulados de Bilbao y Lima, volvieron a oponerse. A lo que Ugarte y Erezcano respondieron, planteando que dichas ordenanzas no eran válidas para puertos sin Consulados ni para Juntas de comerciantes.

El 3 de noviembre, el fiscal Marqués de la Plata llamó a declarar a los antiguos apoderados, para que el Virrey pudiera dictaminar la nulidad o aprobación de la elección. Un mes después, Sarratea y Rodríguez de la Vega aseguraron, 1) que la citación a la Junta se hizo en los mismos términos que todas las anteriores; 2) que el día 20 no se procedió a votar por el escaso número de comerciantes que había; 3) que ese día, Sarratea había solicitado designar un día para la realización de otra Junta, para avisar mediante esquelas, pero la Junta decidió que los concurrentes realizarían la convocatoria verbalmente; 4) que por este motivo, y por estar de antemano invitados para el día 20, se omitió el envío de las esquelas; 5) que el crecimiento de la cantidad de comerciantes, que pasó de 36 a 86 muestra que la tarea se cumplió correctamente; 6) que la escasa participación de los comerciantes en las juntas regulares muestra su escaso interés, no siendo extraño que algunos no asistieran incluso cuando se los citaba; 7) que era cierto que faltó un voto al momento del recuento; $y, 8)$ que hasta ahora las Juntas se habían hecho "sin estas turbulencias ni parcialidades", lo que da cuenta que estamos en presencia de un salto cualitativo en la conflictividad entre comerciantes porteños.

Tal vez por la novedad del enfrentamiento, pareciera que los apoderados no tuvieron por objetivo ni sostener ni anular la elección, intentando permanecer al margen de ambas facciones. Por un lado, alegaron que no podía probarse la inhabilitación de los comerciantes que votaron y que Miguel Larrea, aunque aceptaban que "padeció algunas alteraciones de sentido", se encontraba sano al momento de votar. $Y$, por otro, sostuvieron el pedido de quienes buscaron anular la elección, solicitándole, al Virrey, que eligiese a cuatro individuos para que confeccionasen el listado de los comerciantes autorizados a votar en las Juntas.

${ }^{21}$ Es curioso que este nombre no aparezca como uno de los comerciantes partícipes de la Junta. 
Es difícil diferenciar el discurso de los apoderados de sus verdaderas intenciones. De lo que no parecen quedar dudas es de su objetivo principal: evitar una profundización del enfrentamiento y reconocer que ambas partes tenían parte de razón en los reclamos. Sin embargo, objetivamente, su intervención ambigua parece haber permitido el triunfo de los querellantes: quienes se opusieron a la asunción de Aguirre y Azcuénaga lograron su objetivo, ya que Martín de Sarratea, Manuel Rodríguez de la Vega y Bernardo Sancho Larrea continuaron en su cargo hasta la creación del Consulado.

\section{Conflictos en el interior del Cabildo porteño}

Hemos dicho que uno de los argumentos señalados para detener el nombramiento de Azcuénaga como apoderado del comercio, fue su cargo en el Cabildo de Buenos Aires. Veamos entonces, si en aquella institución se replicaron, de alguna manera, los conflictos entre los comerciantes. Vayamos al mismo año de 1789, en que se desarrolló el enfrentamiento por la elección de los apoderados.

El Cabildo de 1789 lo integraron el Alcalde de primer voto, Miguel Sáenz, el de segundo voto, Miguel de Azcuénaga, los regidores Miguel Mansilla, Diego Mantilla y los Ríos, Gregorio Ramos Mejía, Francisco Ignacio de Ugarte, Diego de Agüero, Benito González Rivadavia, Agustín Casimiro de Aguirre, Cristóbal de Aguirre, José Ramón de Ugarteche, Francisco de Mata y Bustamante, y el escribano Pedro Nuñez ${ }^{22}$. Es decir, tenemos entre ellos a varios de los mercaderes que dinamizaron los conflictos reseñados hasta el momento.

Entre otras numerosas tareas, los capitulares tenían en sus manos cuestiones protocolares, la mayoría de las veces en torno a festividades religiosas y cívicas. De hecho, en la organización de cuestiones supuestamente alejadas del ámbito económico-social, se hacen evidentes allí las diferencias en gestación. Veamos esto más de cerca.

El 8 marzo de 1789, Diego de Agüero se enteró de la "amarga muerte de nuestro Soberano", y fue él quien propuso al Cabildo colocar un retrato de Carlos III en la sala capitular ${ }^{23}$. Junto a Agustín Casimiro de Aguirre, ambos agradecieron al Virrey la autorización del homenaje ${ }^{24}$. Posteriormente Agüero, junto con Estanislao Zamudio, se hizo cargo de organizar las exequias del Rey español, en la Iglesia del Real Colegio de San Carlos ${ }^{25}$. La muerte de Carlos III y la coronación de Carlos IV fue aprovechada por el Cabildo para renovar su fidelidad a España, expresada en la confección de un nuevo "Estandarte de

\footnotetext{
${ }^{22}$ AGN. Acuerdos del Extinguido Cabildo de Buenos Aires. Serie III. Tomo IX. 1789-91, Bs. As: KRAFT Ltda, 1931, pp. 9-12.

${ }^{23}$ Carta de Diego de Agüero a Joaquín Obregón Ceballos, 16 de marzo de 1789. MHN, AH FG SC 07.

${ }^{24}$ AGN, Acuerdos del Extinguido Cabildo, op. cit., p. 136.

${ }^{25}$ Idem, pp. 169-173.
} 
terciopelo carmesí, con las armas reales por un lado, y por el otro con las de la ciudad, pintadas en un lienzo de tafetán", financiado por un préstamo del comercio porteño ${ }^{26}$. El 8 de agosto de 1789, el Cabildo victoreaba la proclamación del "augusto monarca, el señor Don Carlos IV (que Dios guarde) por Rey de Castilla y de las Indias (...) con toda aquella pompa y magnificencia que se acostumbra en estos actos"127.

Pero lo más interesante de esta tarea "protocolar" es que nos permite aproximarnos a los intereses materiales que se esconden detrás de los cabildantes y sus propuestas. Dos grupos debatieron sobre cómo financiar los festejos por el traspaso del trono: uno liderado, una vez más, por Miguel de Azcuénaga; y el otro, por Diego de Agüero. Azcuénaga propuso establecer una escala, para gravar a las diferentes mercancías según ella. Desde su posición, entre los productos que más deberían gravarse se encontraban el cobre, el hierro y el acero (que pagarían dos reales) y, entre los que menos, se destacaban los cueros y la yerba mate (que abonarían un cuartillo), exceptuando de todo pago al "trigo, harinas, carnes, lanas, oro, plata, en pasta o amonedado, por ser unos frutos, y especies de necesidad primera que no admiten gravamen alguno" ${ }^{\prime 28}$. Por el contrario, Diego de Agüero y Agustín Casimiro de Aguirre propusieron gravar "los ramos ya de conocido vicio, y ya de lujo como son el aguardiente, licores, vino, azúcar, miel, dulces, cacao, chocolate, café y yerba mate".

Tenemos, entonces, un enfrentamiento que, como veremos más adelante, se encuentra determinado por las actividades comerciales desarrolladas por cada grupo, y por sus concepciones en torno a los sectores que debían sostener el homenaje al Rey. Por un lado, Agüero rechazó la propuesta de Azcuénaga debido al fuerte gravamen sobre el cobre, una de las mercancías que más traficaba, por el escaso gravamen impuesto a los cueros (giro en el que se especializaba Azcuénaga), y por la enorme cantidad de bienes gravados que eran parte del giro monopolista. Por el contrario, Agüero y Aguirre, al proponer que se graven solamente los "ramos ya de conocido vicio", intentaban descargar en los productos americanos, y en los artesanos, peones y esclavos, el mayor peso del financiamiento. Sin embargo, no lograron imponer su posición, y fueron derrotados.

Ambos grupos, también, tuvieron diferencias en torno al vendaje del pan, que era un impuesto que pagaban los panaderos a los pulperos: por cada peso de pan vendido, el pulpero se quedaba con un real (llamado "vendaje"), como premio a su venta. Los dos bandos se opusieron al proyecto de Tomás Villota, que proponía imponerle al pan este gravamen, además de reducir los puestos de venta. Sin embargo, mientras que Agüero y Casimiro de Aguirre, basados en un dictamen anterior de Manuel Joaquín de Zapiola, José Martínez de Hoz, Manuel de Arana, Miguel González de Noriega y Joaquín Pinto, propusieron gravar especias "de conocido vicio, como el aguardiente, licores, vino, azúcar, miel, dulces, cacao, chocolate, café y yerba mate", Miguel de Azcuénaga,

\footnotetext{
${ }^{26}$ Idem, p. 111.

27 En octubre de 1790, se decide para celebrar "la gloriosa exaltación al Trono de nuestro Augusto Soberano el Señor Don Carlos IV", empedrar las calles de la Ciudad, AGN, Sala IX, Consulado, Expedientes, 1771-1793, Expediente $\mathrm{N}^{\circ} 16,4-7-3$.

${ }^{28}$ AGN, Acuerdos del Extinguido Cabildo, op. cit., pp. 160-161.
} 
Cristóbal de Aguirre y el resto de los capitulares plantearon que había que hacer lo propio con los "renglones que sólo sirven para mantener vicios, o para ostentar el lujo que no contribuyen cosa alguna", imponiendo finalmente su argumentación para el rechazo ${ }^{29}$.

Como podemos ver, comienza a observarse cierta regularidad en la composición de los grupos enfrentados, y encontramos en el ámbito de su reproducción material una clave fundamental para explicar los motivos de su actuación conjunta. En ese sentido, pareciera que, en ocasiones, ese criterio es tenido en cuenta, incluso a la hora de encargar tareas compartidas a ciertos individuos, teniéndose en cuenta su afinidad para el éxito de ciertos encargos. Tal fue el caso de la expedición a salinas, encargada por el Cabildo a Diego de Agüero y Agustín Casimiro de Aguirre (aliados en los debates mencionados) ${ }^{30}$.

Finalmente, estos bandos organizados comenzaban a consolidar un personal permanente y vinculado con cierto grado de organicidad, lo que puede observarse en las votaciones realizadas al finalizar el período del cargo (que era de un año), cuando los capitulares salientes elegían a sus reemplazantes. Fue así como, el $1^{\circ}$ de enero de 1790, Diego de Agüero apoyó la propuesta de Miguel Sáenz, votando como alcalde de primer voto a Pedro Alvarado, como alcalde de segundo voto a José Martínez de Hoz, como regidores a Miguel de Tagle, Juan Agustín de Videla, Martín de Álzaga, Juan de Echenique, Tomás Fernández y Juan de Viola y como síndico procurador a Manuel del Cerro. Sin embargo, a pesar del apoyo de Agustín Casimiro de Aguirre, el voto del resto de los cabildantes modificó sustantivamente esta propuesta ${ }^{31}$. De los 9 capitulares propuestos por Agüero y Aguirre quedaron 6, quedando afuera Pedro Alvarado, José Martínez de Hoz y Tomás Fernández ${ }^{32}$. Aunque podría pensarse que ducha facción logró imponer su línea, el resultado se asemeja más a un empate, ya que los cargos principales (los alcaldes), fueron impuestos por el grupo liderado por Azcuénaga.

\section{¿Aleatoriedad de la acción política? Los intereses en pugna}

Una somera revisión de los escasos, pero significativos, conflictos analizados, nos ofrece un primer elemento a ser señalado: no estamos frente a

\footnotetext{
${ }^{29}$ Idem, pp. 139, 145-147, 151, 162.

${ }^{30}$ No está de más precisar que Susan Socolow se equivoca al afirmar que Diego de Agüero participó de una expedición en 1789, y mucho menos que la financió él mismo de su peculio. Ese año ni siquiera se realizó expedición alguna, como sí se hizo en 1788, a cargo de Agustín Antonio Erezcano y Felipe de Castilla; en 1790, emprendida por Diego Mantilla y los Ríos y Miguel García de Tagle; y en 1791, a cargo de José Martínez de Hoz y Casimiro Francisco de Necochea. AGN, Acuerdos del Extinguido Cabildo, op. cit., p. 104; 108; 114; 165-166; AGN, Sala IX, Cabildo de Buenos Aires, Archivo, 1792, 19-4-5 y SOCOLOW, Los mercaderes..., op. cit., p. 77.

${ }^{31}$ AGN, Acuerdos del Extinguido Cabildo, op. cit., p. 236.

${ }^{32}$ Idem, p. 238.
} 
bandos aleatorios, sino que los choques parecen repetir, a uno y otro lado, figuras preponderantes que se enfrentan en diferentes batallas.

De un lado, es evidente la importancia de la figura de Miguel de Azcuénaga, junto con la de Cristóbal de Aguirre y Francisco Ignacio de Ugarte. Del otro, se repiten los nombres del círculo más cercano a la representación del Consulado de Cádiz en Buenos Aires: Agustín Casimiro de Aguirre, Casimiro Franscisco de Necochea y Diego de Agüero. No obstante, dado que nos encontramos frente a una contradicción que aún no se ha desplegado en el tiempo, no nos es posible encontrar, en los conflictos analizados, una contradicción plena, sino tan sólo enfrentamientos que dan cuenta de diferencias, más o menos sustantivas, que van a desplegarse al calor de la agudización de la crisis del Imperio español tardío. Acerquémonos a dicho proceso, entonces, mediante un breve repaso del derrotero sufrido por los protagonistas de estas trifulcas.

Miguel de Azcuénaga nació en Buenos Aires el 4 de junio de 1754, en el seno de una poderosa familia de comerciantes ${ }^{33}$. Hijo del comerciante español Vicente de Azcuénaga y Rosa de Basavilbaso, durante su niñez fue enviado a cursar estudios en España. A su regreso, en 1773, se alistó en el ejército, iniciando su carrera como subteniente de caballería. Peleó contra los indígenas y los portugueses de la Banda Oriental. Se casó, en 1795, con su prima, Doña Rufina de Basabilbaso, hija del comerciante y administrador general de Correos, Manuel de Basabilbaso. Previamente, como ya dijimos, ocupó varios puestos en el Cabildo: en 1783, fue regidor y alférez real; en 1789, alcalde de segundo voto; $y$, en 1793-94, procurador peneral.

No obstante, una serie de elementos profundizaron la oposición de Azcuénaga con los intereses de su familia, primero, y con los del Imperio español, después. Por un lado, entre 1796 y 1800 se convirtió en el jefe de las milicias y de la guarnición de Buenos Aires. En aquel entonces, se enfrentó a la corporación mercantil, al encarcelar al apoderado Manuel Crespo, quien tenía como misión (junto a Francisco Llano) obtener la gracia para que los mercaderes no tuviesen la obligación de participar del Batallón de Milicias Urbanas del Cuerpo de Comercio ${ }^{34}$. Por el otro, como comerciante legalmente habilitado de la carrera de Indias, comenzó a especializarse en el tráfico de cueros, convirtiéndose en uno de los principales cargadores del puerto de Buenos Aires. ${ }^{35}$ Dicha posición comenzó a vincularlo con los hacendados, alejándolo aún más del núcleo duro de comerciantes monopolistas.

Dicho conflicto se expresó a mediados de 1792, cuando el Rey otorgó un permiso a naturales y extranjeros para realizar el comercio de esclavos, lo que implicaba la posibilidad de extraer frutos (cueros, principalmente) y dinero a

\footnotetext{
${ }^{33}$ SENOR, Maria Selva. 'El azar y la necesidad': élite y elecciones matrimoniales en Buenos Aires 1776-1820. Las familias Azcuénaga y Andonaegui. En: Andes, N 16, Salta, enero/diciembre de 2005.

${ }^{34}$ La Corona les solicitaba a los comerciantes que prestasen su dinero para financiar el combate contra los enemigos de España y, además, que participen, como todo ciudadano, de las milicias dispuestas a defender la Patria. Sin bien los mercaderes siempre estuvieron dispuestos a realizar importantes aportes a las finanzas reales, no fue tan sencillo hacerlos incorporarse a las milicias. AGN, Consulado de Buenos Aires, Legajo 3, № 10.

35 DOUGLASS, William; URZA, Carmelo; WHITE, Linda y ZULAIKA, Joseba (Ed.): La diáspora vasca, Universidad de Nevada, Reno, 1999.
} 
puertos extranjeros. El 17 de julio de ese año, una Junta de Comercio delegó a Miguel de Azcuénaga, Diego de Agüero, Martín de Álzaga, Casimiro Francisco de Necochea, Martín de Sarratea y Manuel Rodríguez de la Vega la tarea de representar al virrey la posición del comercio $^{36}$. Sin embargo, profundas desavenencias existieron entre los delegados, por lo que el informe nunca se presentó. En septiembre, fue reclamado por el Virrey, pero no aparece nunca en el expediente, que pasa de los eventos de 1792 a 1794.

Aunque no tengamos pruebas documentales, es probable que el enfrentamiento haya girado en torno a la exportación de cueros a puertos extranjeros, actividad que Azcuénaga miraba con simpatía, y que los monopolistas no estaban dispuestos a permitir (por lo menos, en aquel entonces). Luego del conflicto, en marzo de 1794, el predominio de la fracción monopolista alejó a Azcuénaga de los intereses más duros del capital mercantil español ${ }^{37}$.

Pasado esto, Azcuénaga estrecha sus vínculos con los hacendados, y llega a convertirse en su apoderado en un momento clave, como lo fue noviembre de 1809, cuando los ingleses solicitaron comerciar con Buenos Aires y el virrey abrió una consulta para tratar dicho ofrecimiento. En aquella situación, la influencia de los sectores que pretendían abrir el comercio porteño a los mercados extranjeros permitió la aprobación del comercio provisional del 6 de noviembre de 1809, exactamente durante la tercera reunión de la Junta consultiva, en la que Juan José Castelli y Miguel de Azcuénaga representaban la posición de los hacendados ${ }^{38}$. Evidentemente, Azcuénaga estaba enfrentándose a los límites que le imponía el sistema colonial.

Su acercamiento a posiciones cada vez más radicales llevó a Azcuénaga a quebrar la tradición monárquica de la familia, distanciándose del programa de

\footnotetext{
${ }^{36}$ Participan de la Junta, además de los comisionados, Jayme Alsina y Verjés, Bernardo Gregorio de las Heras, Joaquín de Arana, Esteban Villanueva, José González de Volaños, Antonio de las Cagigas, Domingo Belgrano Pérez, Agustín Wright, Francisco Antonio de Escalada y Pedro Duval, entre otros. AGN, Sala IX, Consulado, Expedientes, 1771-1793, Expediente $N^{\circ}$ 22, 4-7-3.

37 Una Junta de 31 comerciantes delegó su representación en Diego de Agüero, Martín de Álzaga y José Martínez de Hoz, quienes protestaron al Virrey por "los gravísimos perjuicios y atrasos que resultarán al mismo Cuerpo y a la Real Hacienda del efecto de la Real gracia obtenida por Tomás Antonio Romero, para extraer desde aquí en derechura a dominios extranjeros el importe de 250.000 pesos en frutos del país". Entre los solicitantes figuraban Francisco Castañón, Juan Vilardebo, Juan Viola, Joaquín de Arana, Antonio de las Cagigas, Pedro Duval, Miguel Antonio Cornet, Francisco de la Peña Fernández, Antonio García López, Gaspar de Santa Coloma, Juan José Lezica, Isidro José Balbastro, Jaime Alsina y Verjés, Francisco Ignacio de Ugarte, Tomás Fernández, Casimiro Francisco de Necochea, Cristóbal de Aguirre, Miguel González de Noriega, Andrés de Lista, Francisco Antonio de Escalada, Bernardo Gregorio de las Heras, Juan Antonio de Lezica, José Ramón de Ugarteche y Francisco Antonio de Beláustegui. AGN, Sala IX, Consulado, Expedientes, 1771-1793, Expediente $\mathrm{N}^{\circ} 22,4-7-3$.

38 Naturalmente, no lograron solos dicha hegemonía. En la junta consultiva también se destacaron otros hacendados, quienes aparecían como representantes de otras instituciones: el capitular Manuel Obligado, el comandante de Patricios, Cornelio Saavedra y el consular Francisco Antonio de Escalada. AGI, Buenos Aires, 589.
} 
su padre ${ }^{39}$. Fue un fervoroso defensor de la Revolución de Mayo de 1810, siendo nombrado vocal de la Junta de 1810. Desde su cargo, enfrentó a los realistas, cortando toda comunicación con Montevideo y destituyendo a los miembros del Cabildo de Buenos Aires ${ }^{40}$. La transgresión de sus medidas, implicaban la muerte, incluso para individuos vinculados a su familia, tal como aseguraba, en diciembre 1810, el comandante realista José María Salazar, al referirse al fusilamiento, en la plaza principal de Buenos Aires, de Basilio Viola y Juan González:

por haber encontrado una carta que escribían al comandante del bloqueo reclamando una lancha detenida, fueron pasados por las armas a pocas horas de presos, sin forma de proceso, ni oírlos, ni darles más tiempo que para confesarse, no habiéndole servido, al primero, ser pariente del vocal Azcuénaga, y su mujer del vocal Belgrano y, al segundo, ser un viejo de más de setenta años ${ }^{41}$.

En 1812, el mismo día del ajusticiamiento de Martín de Álzaga y sus aliados, que habían intentado dar un golpe contrarrevolucionario, Miguel de Azcuénaga requisó todas las "armas de chispa o blancas" a los españoles, expropiando a los viejos comerciantes con los que, otrora, compartió juntas y reuniones, como José Martínez de Hoz, Juan Antonio de Santa Coloma, Manuel Ortiz de Basualdo, Esteban Villanueva, Francisco Beláustegui, Tomás Antonio Romero, Antonio de las Cagigas y Anselmo Sáenz Valiente ${ }^{42}$.

La radicalización de Azcuénaga no tuvo equivalente en los aliados de sus primeros tiempos, Cristóbal de Aguirre y Francisco Ignacio de Ugarte. No obstante ambos estuvieron vinculados al tráfico con mercados extranjeros, y buscaron superar el intercambio restringido entre Cádiz y el Río de la Plata ${ }^{43}$, ambos han oscilado entre las dos fuerzas sociales a lo largo de su vida, como lo prueba su participación en las juntas que buscaron detener la gracia otorgada a Tomás Antonio Romero. No obstante, hay que destacar que la vinculación de Aguirre con los hacendados llegó, incluso, al plano familiar, en tanto fue padrino

\footnotetext{
${ }^{39}$ Poco antes de su muerte, Vicente de Azcuénaga solicita al Virrey que anule el trámite de mayorazgo que había realizado a favor de su hijo Miguel. A su vez, designa a su yerno, el monopolista Gaspar de Santa Coloma, como primer albacea y tutor de sus hijos menores. Descontando el enojo de Miguel, su padre advierte que "si en la prosecución del pleito el referido Don Miguel, mi hijo, se excede en palabras y razones injuriosas contra mi honor, fama y buena reputación [...] es mi voluntad desheredarlo". LESSER, Ricardo. La infancia de los próceres. Buenos Aires: Biblos, p. 170.

${ }^{40} \mathrm{El} 1^{\circ}$ de agosto, Miguel de Azcuénaga y Mariano Moreno advirtieron, al capitán del puerto de Buenos Aires, que "por ningún motivo obedezcan disposición alguna del comandante de marina de Montevideo, y que se mantengan enteramente a disposición de la Junta". AGN, Sala III, Capitanía del Puerto, 1810-1813, Caja 1.

${ }^{41}$ Este testimonio confirma lo endeble de las interpretaciones que consideran los lazos familiares como los determinantes centrales de las alianzas políticas. AGI, Leg. 123-2-4, citado en WILLIAMS ÁLZAGA, Enrique. Álzaga, 1812. Buenos Aires: Emecé Editores, 1968, pp. 242-243.

${ }^{42}$ Archivo de la República Argentina: Antecedentes políticos, económicos y administrativos de la Revolución de Mayo de 1810, Buenos Aires, Tomo XI, p. 278, 1924.

${ }^{43}$ SAGUIER, Eduardo. Colapso del entrepôt mercantil y lucha en el hinterland contra el monopolio comercial. En: Genealogía de la Tragedia Argentina, versión on-line en http://www.er-saguier.org [consulta el 20/1/2010].
} 
de nacimiento de José Francisco de San Martín (el futuro "padre de la Patria"), y padrino del casamiento de Juan Esteban de Anchorena, vinculándose además con Manuel Obligado ${ }^{44}$. También fue, en los momentos en que militaba en el grupo de Azcuénaga (hacia 1789), apoderado del comerciante de Colonia de Sacramento, el portugués Manuel Acuña Nebes, vínculo que lo estrechaba con mercados extra-españoles ${ }^{45}$. Finalmente, como síndico del Consulado de Buenos Aires (1794 y 1796), se desempeñó como un "ardiente librecambista", al decir de Germán Tjarks ${ }^{46}$.

Del otro lado, sus oponentes desarrollaron una radicalización similar a la de Azcuénaga, aunque defendiendo un programa antagónico. El grupo de comerciantes vinculados al Consulado de Cádiz en la década de 1780, Casimiro de Aguirre, Necochea y Agüero, no sólo permanecieron unidos hasta su desaparición física, sino que también dinamizaron, junto a otros de los principales monopolistas rioplatenses, como José Martínez de Hoz, Jayme Alsina y Verjés y Martín de Álzaga, el corazón de la resistencia al proceso de transformación social que atravesó el Río de la Plata a principios del siglo XIX ${ }^{47}$.

Aunque ese proceso ya ha sido estudiado en otros trabajos, nos basta con señalar aquí como elemento central el hecho de que Agustín Casimiro de Aguirre era, en la década de 1770, apoderado del Consulado de Cádiz ${ }^{48}$. Su

${ }^{44}$ CAULA, Elsa Stella Maris. Parentesco, amistad y paisanaje: los vascos en el Río de la Plata. Sancho el sabio, No 12, 2000, pp. 131-154.

45 TEJERINA, Marcela. Luso - brasileños en el Buenos Aires virreinal. Trabajo, negocios y e intereses en la plaza naviera y comercial. Bahía Blanca: Universidad Nacional del Sur, pp. 193 y 227, 2004.

46 TJARKS, Germán. El consulado de Buenos Aires y sus proyecciones en la historia del Río de la Plata. Bs. As: UBA-FFyL, 1962, p. 294. El 12 de junio de 1807, Aguirre volvió al tribunal comercial, esta vez como segundo cónsul. El 10 de junio de 1808, ascendió a primer cónsul y, el 16 de junio de 1809, pasó a ocupar el puesto de consiliario, donde aún permanecía en mayo de 1810. Luego de la Revolución, continuó en su cargo, siendo reemplazado el 12 de septiembre de 1811, por una nueva Junta nombrada por decreto por el gobierno revolucionario. Ocupó, además, una numerosa cantidad de funciones políticas y burocráticas: fue juez, oficial real, vocal, regidor perpetuo y alcalde de primer voto del Cabildo de Buenos Aires. El 31 de diciembre de 1802, el Cabildo propuso al Rey Carlos IV que nombrase a su Ministro el Príncipe de la Paz, Manuel Godoy, "Regidor honorario del Cabildo de Buenos Aires". Luego de que el Rey accediese a la solicitud, en 1804, Cristóbal de Aguirre fue elegido para servir en el puesto "a nombre del Excelentísimo Señor Príncipe de la Paz", acordándose que tendría voz y voto en las deliberaciones. También participó de las milicias: en 1795 le fue concedido el retiro con el grado de Capitán de Infantería de las milicias de Buenos Aires. Sin embargo, al producirse las Invasiones Inglesas al Río de la Plata, en 1806 y 1807, ya anciano, combatió como coronel del Tercio de Vizcaínos. En tanto vecino principal del comercio de la ciudad, fue invitado, junto a su hijo Juan Pedro y su yerno Gerardo Bosch y Alvareda, al cabildo abierto del 22 de mayo de 1810, aunque se retiró sin emitir su voto. Falleció en la ciudad de Buenos Aires el 11 de junio de 1831, a los 95 años.

47 SCHLEZ, Mariano. Enemigos declarados de nuestro sistema. La acción política contrarrevolucionaria de Diego de Agüero y Miguel Fernández de Agüero (1770-1816). En: Anuario del Instituto de Historia Argentina, Facultad de Humanidades y Ciencias de la Educación - UNLP, No 11, 2011.

${ }^{48}$ Carta de Salvador de Trucios a Diego de Agüero, ausente Agustín Casimiro de Aguirre, 26 de mayo de 1774, AGN, Sala VII, Legajo 761, Foja 17. 
muerte dio lugar a la asunción, como apoderado gaditano, de Casimiro Francisco de Necochea, quien posteriormente delegó el cargo en Miguel Fernández de Agüero, sobrino y principal socio de Diego, en 1804. En efecto, fue Fernández de Agüero el encargado de enfrentarse a quienes fomentaron la apertura mercantil a los ingleses, en 1809, entre ellos a Miguel de Azcuénaga y Mariano Moreno ${ }^{49}$.

\section{Del combate larvado a la guerra abierta: los antecedentes de la Revolución de 1810}

Comprender la naturaleza de los enfrentamientos que dieron origen a los Estados nacionales en América Latina implica un conocimiento fino, tanto de los bandos enfrentados, como de los fundamentos de su actuación política y militar. Ello implica que el análisis de las fuerzas opuestas requiere de una mirada que supere una mirada estrecha, y vaya más allá de la coyuntura específica de $1810^{50}$.

En ese sentido, hemos podido comprobar que, en el caso de la revolución rioplatense, los mismos protagonistas de los combates revolucionarios decimonónicos, organizados en bandos perfectamente reconocibles, comenzaron a profundizar sus diferencias de manera cada vez más sistemática, cuarenta años antes del estallido independentista. Azcuénaga, Aguirre, y Ugarte, por un lado, y Agüero, Necochea y Casimiro de Aguirre, por el otro, aparecen como los referentes de un conjunto mayor de individuos que se colocaron tras su dirección, y que poseían posiciones enfrentadas en torno a diferentes coyunturas y problemas específicos.

Su unión no parece haberse basado en aspectos meramente subjetivos, sino que encuentran su raíz en el tipo de actividad mercantil que cada uno desarrolla, es decir, en la posición social que poseen en la estructura económica colonial. Es así como cada bando defiende sus intereses, desde los combates más minúsculos, como lo puede ser la financiación de los funerales del Rey, hasta los más fundamentales, como el nombramiento de apoderados del comercio, basado en sus intereses materiales.

No obstante, como hemos intentado probar en el último apartado, la naturaleza de esos enfrentamientos no puede ser explicada restringiéndose a esos encuentros específicos, sino que debemos avanzar en el paso del tiempo para observar el despliegue de las contradicciones y la evolución de las alianzas. Es así como, observando el derrotero de los comerciantes en cuestión, comprendemos que aquellas primeras reyertas expresaban, de manera larvada, un antagonismo entre el grupo vinculado al Consulado de Cádiz, que busca controlar más férreamente el tráfico colonial y mantenerlo en manos exclusivamente españolas, frente a aquellos comerciantes interesados en

\footnotetext{
${ }^{49}$ SCHLEZ, Mariano: Está muy cercana la subversión... La lucha política, económica y militar en el Río de la Plata (1809). En: Actas del I Congreso Internacional sobre Temas Americanistas, Revista Temas Americanistas-Universidad de Sevilla, Sevilla, España, 8 al 10 de noviembre de 2012.

${ }^{50}$ HARARI, Fabián. En busca del Sujeto revolucionario: las vaquerías en Buenos Aires a comienzos del siglo XVII. Razón y Revolución, n 10, primavera de 2002.
} 
exportar frutos a puertos extranjeros, y en desarrollar relaciones de nuevo cuño en el Río de la Plata.

A partir del análisis de ese proceso de mediano plazo, es evidente que el proceso de lucha política pasa de una etapa de combate potencial a una de lucha abierta, política y militar, en donde las contradicciones salen a la luz y se hacen más evidentes. Nuestro artículo se concentra en el estudio de esa primera etapa, menos conocida, y más difícil de estudiar por la falta de documentación, mostrando que, incluso en aquel entonces, se observa el enfrentamiento de bandos delimitados en torno a diferencias económico-sociales, las que se ahondarán al calor de la crisis y de la revolución, trasluciendo al carácter clasista de su antagonismo.

\section{Sobre 0 autor}

Mariano Schlez es Doctor en Historia de la Facultadde Filosofía y Letras de la Universidadde Buenos Aires (UBA). Actualmente desarrolla sus estudios posdoctorales en el Área de Historia Americana y Argentina, del Departamento de Humanidades de la Universidad Nacional del Sur, como becario del CONICET (Argentina), dedicándose al estudio del capital mercantil en el Río de la Plata. En la actualidad se desempeña como investigador del Programa de Incentivos del Ministerio de Ciencia y Educación dela Nación(categoría IV), del Centro de Estudios e Investigación en Ciencias Sociales (CEICS) e integra el proyecto "Los Otros en dimensión histórico política: tensiones, conflictos y dinámicas de poder en el Río de la Plata (fines del siglo XVIII y principios del XIX)" (Universidad Nacional del Sur). En la UBA, es docente regular de la carrera de Historia (Facultad de Filosofía y Letras), desempeñándose como profesor en la cátedra Didáctica Especial y Prácticas de la Enseñanza de la Historia. Es autor de numerosos artículos sobre el sistema colonial español y los procesos revolucionarios del siglo XIX, publicados en revistas científicas de Argentina, Brasil, Perú, Colombia y España. Es autor del libro, Dios, Rey y monopolio. La naturaleza social de la contrarrevolución en el Río de la Plata a comienzos del siglo XIX. El caso de Diego de Agüero (1768-1816) (Buenos Aires, Ediciones ryr, 2010). E-mail: mmschlez@hotmail.com

Artigo recebido em 01 de maio de 2014. Aprovado em 15 de junho de 2014. 